\title{
Grégoire Nicolis, of the Founders of Complexity Science: A Recollection
}

\author{
V. Basios* and S. Nicolis ${ }^{\dagger}$ \\ Interdisciplinary Center for Nonlinear Phenomena and Complex Systems (CeNoLi), \\ Université Libre de Bruxelles (ULB), BELGIUM \\ (Received 29 November, 2019)
}

\begin{abstract}
Grégoire Nicolis' memorable and seminal influence in the development of modern statistical mechanics, physical chemistry and nonlinear dynamics, his contribution to founding Complexity Science and memories of his tutelage are presented in this short review. After tracing the guiding ideas of his numerous contributions we discuss two particular and contemporary unfolding areas of research that constitute part of his rich heritage. The first stemmed of his later work on a new paradigm of crystallization and aggregation and has to do with the new developments towards self-organized, adaptive or 'smart' materials. The second is related to his arching interdisciplinary ideas concerning complex systems that, having reached biology, are leading the investigation of coordinated motion and decision making in collective dynamics of social animals.
\end{abstract}

PACS numbers: 05.65.+b,05.45.-a

Keywords: complex systems, complexity, nonlinear dynamics, out-of equilibrium, statistical mechanics, emergence, collective motion, entropy

DOI: https://doi.org/10.33581/1561-4085-2020-23-2-102-112

"The whole is more than the sum of its parts"

Aristotle Metaphysica 1045 a, often quoted by $G$. Nicolis

\section{Introduction}

Grégoire Nicolis' brilliant scientific life came to limelight[1] with the creation, with R. Lefever and I. Prigogine, of a model that now is known as the 'Brusselator' [39] (the 'Brusselator' is treated in Chapter VII). It was one of the first models of chemical kinetics that sustained oscillations via an auto-catalytic, nonlinear, reaction scheme. It played and still plays a central role in our understanding of the roe of nonlinearities in chemical kinetics and paved the way to the

\footnotetext{
${ }^{*}$ E-mail: vbasios@ulb.ac.be; Service de Physique des Systèmes Complexes et Mécanique Statistique, Université Libre de Bruxelles (ULB), BELGIUM

${ }^{\dagger}$ E-mail: snicolis@ulb.ac.be; Unité de recherche en Chimie physique et Biologie théorique, Université Libre de Bruxelles (ULB), BELGIUM
}

discovery of chaos in electrochemical reactions by his friend and long-time collaborator Otto Ros̈sler. These early discoveries in chaos theory constitute part of its foundations as they brought forth deep connections of nonlinear dynamics and out-of-equilibrium processes in physics and chemistry at large. Especially, Grégoire Nicolis' with his prime student Pierre Gaspard shed light, early in the history of complex systems in statistical mechanics, on the role of topological aspects of dynamical systems for the appearance of non-trivial steady states out of equilibrium, known now as chaotic/strange attractors or 'dissipative structures', and discovering the role of homoclinic connections (e.g. Shilnikov's connection and Smale's Horshoe structure), as one of the main route to chaos [2].

Since these early days the scope and purview of Grégoire Nicolis' work towered spreading over what now we understand as Complex System's Science, or 'Complexity'. His publications span an vast array of subjects in a truly inter-disciplinary and trans-disciplinary fashion. Estimated in about 300 papers in peer reviewed journals, 13 edited volumes and five books, translated in various languages, his scientific work is not 


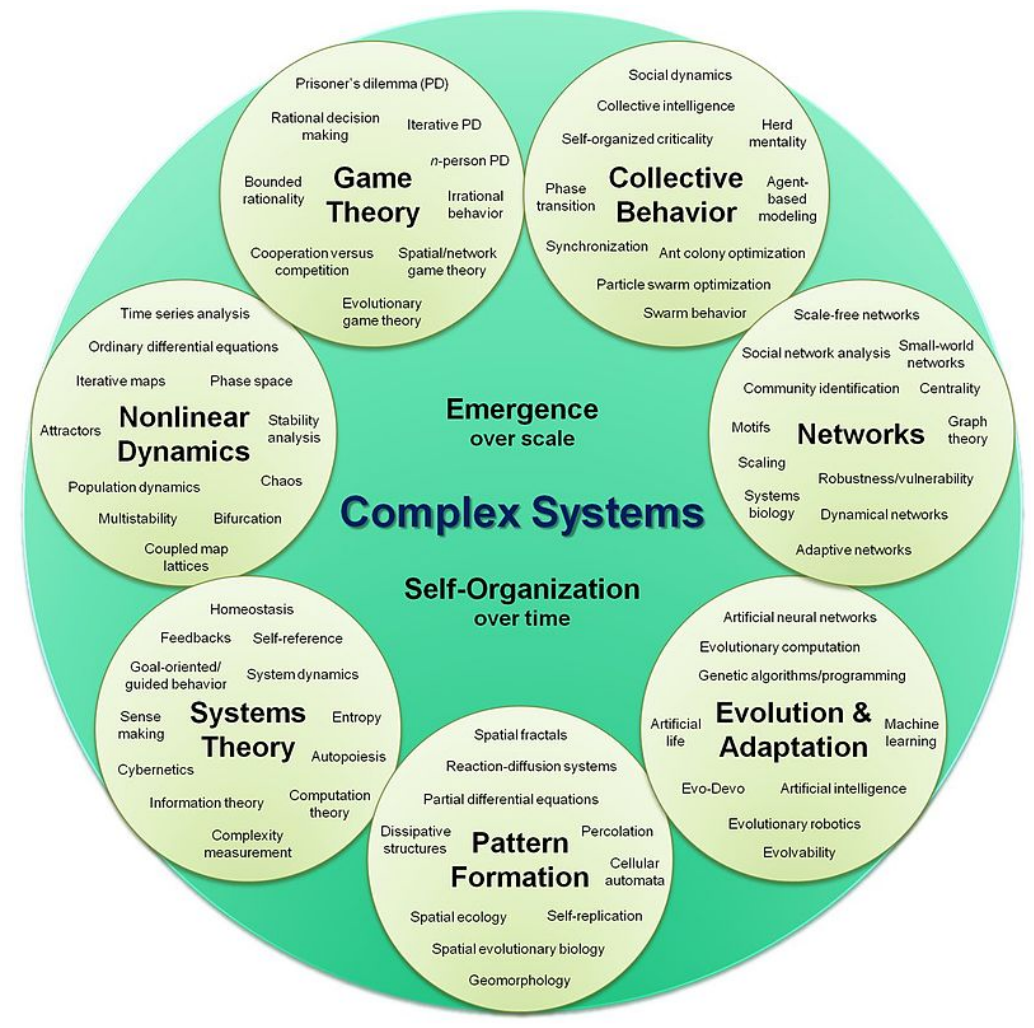

FIG. 1: (color online) Complex System's organizational map [4].

limited to just academic publishing as a member of many key organizations, notably European, Belgian and Greek Academies of Sciences, and a plethora of consulting bodies he first heralded and then continued to support and promote the science of complexity throughout his life until his sudden and untimely passing. Actually, just a few months before he passed away he published his last work, the introductory chapter for a book on the foundations of complexity [3]; there, one can hear him discussing his personal account on the foundations of complexity where he played a pivotal and strategic role in establishing.

Nicolis' wide and deep insight in nonlinear dynamics can be traced to his formative years in the Technical University in Athens, where he obtained a firm basis on applied mathematics and physics. He mastered nonlinear dynamics or 'catastrophe theory' as it was then known the state of the art of the times as well as Carathéodory's theories in thermodynamics. When he joined the group of Prigogine's in Brussels, circa 1963, he was at home within a tradition that combined, high-level mathematics and path-breaking thermodynamics and physical chemistry. His academic heritage includes Théophile Ernest De Donder, of the celebrated 'affinity theory', and stretches back to the great Henri Poincaré. Having assimilated the great works before him himself became a brilliant and sensitive teacher for a long list of graduate students, helped establish graduate schools and study-programmes on complex systems. Some of his students now being themselves directors and professors in the general area of complex systems allover the world. And in Brussels, where the research centre named "Centre for Nonlinear Phenomena and Complex Systems" (or "CeNoLi" for short) that he established in the early 1990's is now run by his own students. CeNoLi was on of the first such centers and at the time of its conception was a truly pioneering concept due to its groundbreaking research orientation. The many and flourishing research centres for complex 
systems that are sprouting nowadays all over the world testify that his vision has reached beyond the horizons of his era.

\section{Seminal \& key contributions}

If we put ourselves to the task of summarizing the life-long achievements of Grégoire Nicolis we can see his influence and heritage to cover these main areas: Firstly he developed further and with his teacher, Prigogine, the theory of open systems and he established the validity of fluctuation dissipation theorem, in relation to the second law of thermodynamics and entropy production.

Then he continued to firmly establish the role of dissipative structures in morphogenesis and thus opened the way for the study of pattern formation in reaction-diffusion systems.

Subsequently he pursued with his team and coworkers a wide investigation in the role of bifurcation and chaos and notably their interplay with stochastic dynamics. As an outcome, he continued and proposed a probabilistic approach to chaos that employed both metric considerations of ergodic theory combined with the topological features of dynamics and gave a new, really regenerative, thrust to symbolic dynamics for complex systems.

His dear wife Cathrine (Kate) Nicolis, his prime partner in his scientific life and life at large since they have been married happily for over than fifty years, they contributed seminal works on the interplay of stochastic and chaotic dynamics, revealing surprising results for the role of noise in nonlinear dynamics. Kate Nicolis studies on the variability of the evolution of the earth's climate led to the discovery of the phenomenon of "Stochastic Resonance" $[5,6]$. A phenomenon that is ubiquitous and generic to any nonlinear system under periodic forcing under noise and/or uncertainty, with extremely important implications for biology and neuroscience in particular. The foundational work of Kate \& Grégoire Nicolis on stochastic resonance and in general the constructive role of fluctuations in nonlinear/chaotic systems, as well as their relations to entropy, symbolic and information dynamics, with the prediction of complex systems, by now, constitutes a classic reference $[35,36]$.

In this context, Kate \& Grégoire Nicolis with their coworkers, Ebeling, J.\&G. Subba Rao, J.S. Nicolis and others have extensively studied the effect role of memory and constraints in the symbolic dynamics of Markov processes arising from the coarse-graining of nonlinear time-series. They studied the information content (Block Entropy) of the one-dimensional, information rich, traces of symbols sequences they conjectured the rise of various, qualitatively different scaling rules, of information measures. What is known as the 'Ebeling-Nicolis conjecture' in symbolic dynamics proved a very fertile ground extending such studies from the study of DNA-symbol sequences, to musical and human texts, to intermittent processes and the presence of the ubiquitous Zipf's law $[7,9,30]$ The role of super-selection rules, memory, history, emerging evolution and their connection to the underlying dynamical aspects, such as feedbacks and hysteresis' loops, to the expression of information dynamics prediction and control of complex systems brought into the foreground the time-dependent properties of the dynamics, or the "information entropy of a history" [35](Chapter 4.4) and [30] (especially Chapter 9, "Selforganization of Symbols and Information" by W. Ebeling and R. Feistel). A theme that another friend of his, G. Chaitin, has developed even in the heart of pure mathematics, proving the infinite irreducible complexity of the halting probability, $\Omega[40]$.

Grégoire Nicolis never ceased to highlight the 'importance of being nonlinear'. He has taught us the constructive role of nonlinear feedback and self-reference (with exemplar the role of auto-catalytic reactions in pattern formation) and provided the very framework of the study of self-organization in complex systems. He was a deep thinker and as such his work has a deeply educational character directly addressing epistemological and philosophical issues related to complex systems [38]. His work has been guiding the research about self-organization and 
emergence in complex systems. But also he has been educating us all of how to think about complex systems. One of the most characteristic passages of his approach is found in his classic textbook, [36], where he states in the introduction:

"... Nonlinear science introduces a new way of thinking based on a subtle interplay between qualitative and quantitative techniques, between topological, geometric and metric considerations, between deterministic and statistical aspects.

It uses an extremely large variety of methods from very diverse disciplines, but through the process of continual switching between different views of the same reality these methods are crossfertilized and blended into a unique combination that gives them a marked added value.

Most important of all, nonlinear science helps to identify the appropriate level of description in which unification and universality can be expected ...".

Spanning an immensely wide array of areas of knowledge contemporary Complex System's Science is one of the fastest growing scientific endeavors. The foundations that Grégoire Nicolis, among the other pioneers of complexity, laid hold strong and firm supporting and inviting fresh approaches and creative thinking for confronting the many open problems and applications at its frontiers. With the advent of modern computational complexity theory, or 'data sciences' and network theories a fusion of classical concepts with modern information techniques is taking place these days.

Since a complex system, by definition, is comprised of many nonlinearly interacting constituent parts, 'a whole which is more that the sum of its parts' like Grégoire Nicolis always loved to refer to, its study raises challenges that go beyond classical reductionist thinking. And as another great scientist of our times, Stephen Hawking, famously and quite 'prophetically' asserted "...the next (21st) century will be the century of complexity" it seems obvious that we are dealing with a wonderful new scientific landscape. Grégoire Nicolis has left us no only firm foundations dealing with complexity but also with an inspiring heritage bearing fruits for the near and far future.

\section{Legacy \& heritage}

The immediate legacy of Grégoire Nicolis is his numerous students spreading in institutions all over the globe. Yet, to try to trace the breadth and width of his influence is beyond the scope of this short review. Actually this is work for future historians of science when they will study the emergence of the science of complex systems. Yet, we can testify here, as his students and close collaborators, the impact and influence of his work in our own and our vicinity. The horizon of which is necessarily limited and partial.

The 'umbrella' research centre, 'Cenoli' that he established where physics, chemistry and biology departments participate was an early pioneer in establishing complex systems research.

In physics, the team that carries his legacy is directed by one of his prime students, Pierre Gaspard [41], a renown leader in statistical mechanics out of equilibrium, molecular machines, theoretical nano-science, the physics of complex systems and active matter.

In chemistry Anne De Wit [42], another of his prime students and leader of her field, is heading the nonlinear physical-chemistry research unit carrying out work work on patternformation in, Turing instability, the spatiotemporal dynamics of patterns that emerge under chemical reactions and hydrodynamic instabilities (chemohydrodynamics) with applications to engineering, nonlinear science and environmental issues.

In biology, one of his earliest and prime students, Jean-Louis Deneubourg [43], is one of the early pioneers in research that established the field of study of collective dynamics in social animals. He is leading a group studying the underlying dynamical basis of self-organization of animal societies in collective decision-making and the study of how these colonies can display cognitive capabilities that go beyond the scope of single individuals.

For an quick overview of Grégoire Nicolis vast heritage, that runs throughout the present- 
day landscape of complexity 1 , one can consult his article in 'Scholarpedia' [44] and for a deeper understanding their book, with Catherine Nicolis, is highly informative for the future directions of the new paradigm of complexity science and its resultant epistemological and wider implications [35] (especially Chapter 6.8).

We now turn to two specific areas of study that we have been directly involved among other and have been inspired from Grégoire Nicolis' latest work in his recent past and last years. The first has to do with a new paradigm of crystallization and aggregation in complex nanomaterials that he and Catherine Nicolis have established and we carry on to smart materials and the second with the complex dynamics underlying decision making in social animals.

3.1. Complex Matter: From a new paradigm of crystallization to advanced, selforganized, materials

In the early 2000s experiments with crystallization of proteins and self-assembly in zeolite synthesis posed challenging questions as to the very nature of their underlying dynamics[10]. Standard nucleation theory did not yield the correct answers for observations of the crystallization of nanoparticle systems (namely proteins and zeolites) under microgravity conditions. It soon became evident that the issue was not a peripheral issue of microgravity physics but the noticed deviations were pointing to a fundamental limitation of classical nucleation theory, at large. So, the group of Grégoire Nicolis' at ULB was called to join the investigation of what is fundamentally related to issues related to the complexity of the materials under observation in the International Space Station Laboratories in space and earthbound [13].

As usual his attention was focused in the crux of the matter, the evidence that this self-organization phenomenon occurs in the presence of a metastable phase, still elusive and uncharacterized at that time but more and more well known and characterized by today. Once the key issue was identified Kate and Grégoire Nicolis provided a simple, actually the simplest possible, generic, model that can capture the effect of this metastable phase in the kinetics of the crystallization pathways [11]. They showed that such an intermediate phase provide an extra pathway that turns on non-standard nucleation mechanisms with combined structural and density fluctuations, as Figures 2 and 3 illustrate.

Once more the influence on kinetic effects of nonequilibrium states was highlighted and draw the attention of a wider community furnishing experimental and simulation-based support for their hypothesis over the subsequent years. An important part of this process is that the effect of metastable phases on the free energy landscape is determined for such classes of nanomaterials with interparticle interactions that are weakly attractive and short-ranged. Their generic model involves two order parameters, the first is the standard crystallization parameter, destiny, while the other is a non-standard order parameter that determines the structure of the material, i.e. crystallinity, number of bonds etc. The introduction of the second order parameter opened the way to accommodate non-standard pathways where the material is firstly selforganized in an intermediate phase and then aggregates to its final solid, crystal form. In Figures 2 and 3, the two pathways are shown: with red the, newly found pathway via a second less structured intermediate phase $(X)$, non-standard one and with blue line the standard Gibbs-like pathway to $S$ with the same structure present throughout. A second order parameter also implies that fluctuations can have an additional structural effect. The important conclusion of that seminal paper was that it identified the conditions under which the transition rates for each pathway can he preferred.

The presence of an intermediate phase had accumulated evidence from experimentalists' groups and was a topic of debate in the early years of its discovery. The theoretical justification by the group of G. Nicolis gave full support and thrust of this idea that proved a pathbreaking one. What accomplished this theoretical breakthrough is was a seemingly simple but rich in implications generic model. Inspired from this approach several other works arose with more detailed models, 
TWO-steps, ONE order-parameter
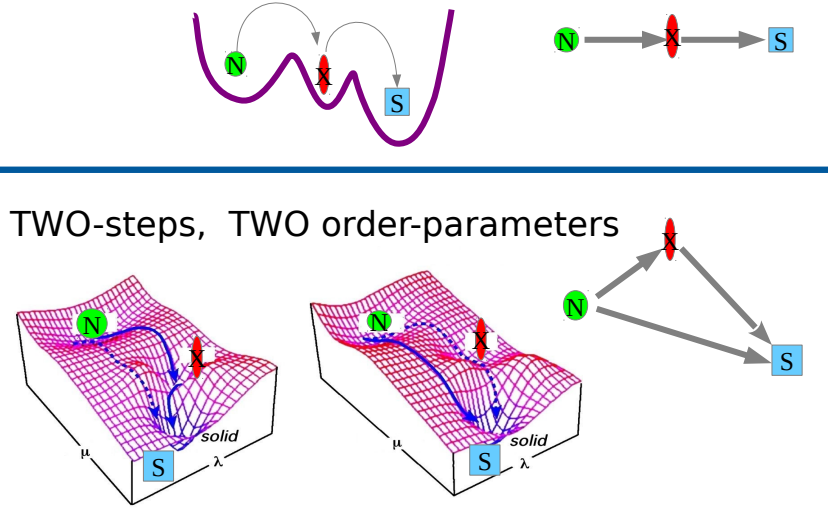

FIG. 2. (color online) An extra order parameter, describing structure, provides a topologically qualitatively different picture of nucleation aggregation allowing extra pathways: The standard one $N \rightarrow S$ upper panel and the non-standard one via $X$-intermediate(s) $N \rightarrow X \rightarrow S$. Where $N$ stands for nanoparticle oligomeres, $X$ the transient intermediate(s) and $S$ the final solid product(s).

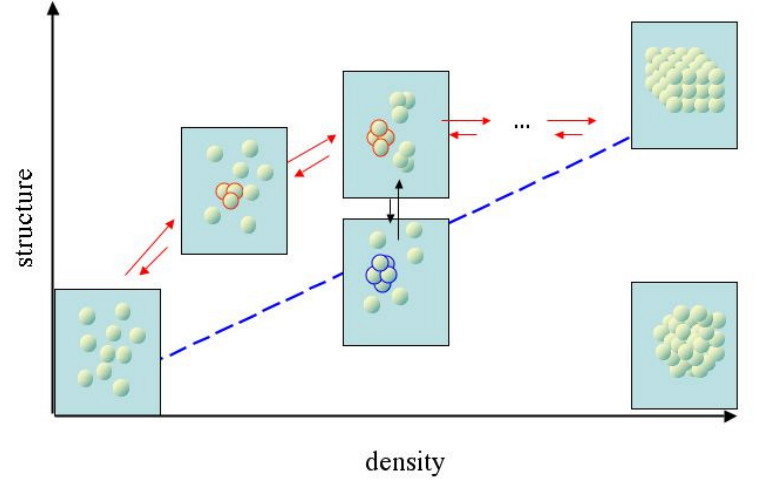

FIG. 3. (color online) The new paradigm of crystallization/aggregation allows different pathways. It predicts the existence of an intermediate phase with variably ordered material of that of the final structure. The new order-parameter that accounts for structure $(y$-axis) permits both structure and density ( $x$-axis) fluctuations. With the blue line is the standard (Gibbs' picture) phase diagram of state transitions and with the red line the new paradigm, involving two (or more) aggregation/nucleation steps via intermediates.

simulations and experimental findings. These have been presented and summarized in [14], for a recent review of the state of the art, see [19] and references within. An interesting area of application of these ideas came about in zeolite synthesis, also coming from migrogravity related experiments, where the intermediates

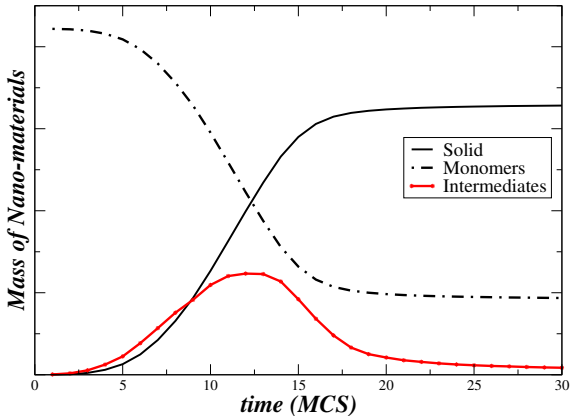

FIG. 4. (color online) Mean field picture of twostep aggregation. The intermediates (red-line) appear, facilitate selforganization and disappear giving way for the final product.

were identified as a population of nano-sized aggregates with various distinct shapes. Once the process is modeled via standard Monte-Carlo techniques different aspects can be revealed. Here are two prevailing features that emerge: The first is the characteristic signature of such a nonstandard aggregation/self-organization process. Figure 4 shows the mean field results of the process of zeolite aggregation via intermediate states, the intermediate population of nano-sized blocks appear, aid the formation of the final product and disappear as they are incorporated in the final self-catalyzed, synthesis. Figure 5 shows the fractal dimension of these intermediate aggregates calculated in various phases as they 
Fractal Dimension Evolution of Nano-materials

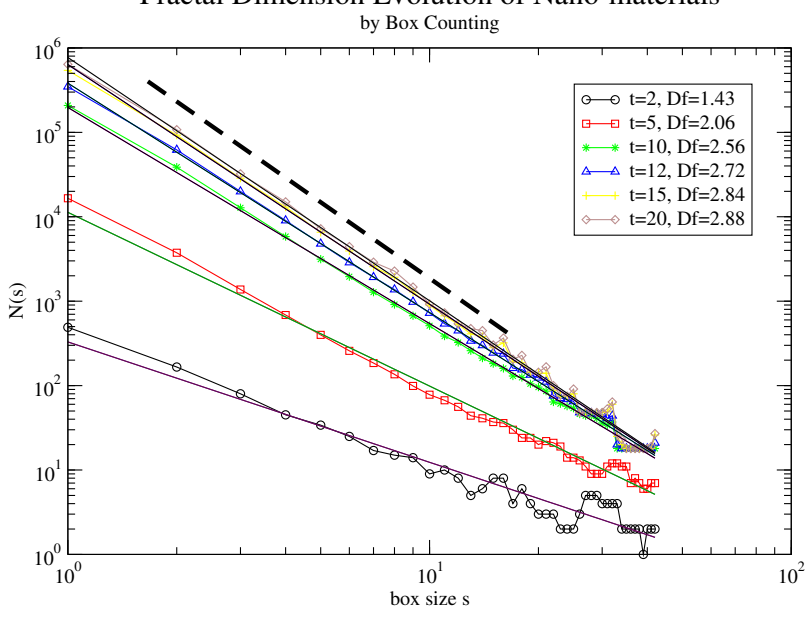

FIG. 5. (color online) The intermediate, metastable state, is made up by pre-embryonic crystal-structures different than the final result; as the evolution of its fractal dimension testifies.

develop in time. They start at time, $t=2$ (measured in 'Monte Carlo Steps') with a fractal dimension $D_{f}(t=2)=1.43$ and gradually arrive about $t=20$ in a fractal dimension $D_{f}(t=20)=2.88$ very close to that of the full grown solid $\left(D_{f}(t \rightarrow \infty)=3\right)$, the broken line in Figure 5. Similar non-standard aggregation processes have been observed also in the context of social-animal collective dynamics. An example coming from J.-L. Deneubourg's team is the step-by-step aggregation in Trophalaxis (food exchange) that optimizes food gathering and storage in the common 'social stomach' of an ant colony as well other goal-optimizing aggregation instances [16, 34]. Complex macromolecular and supramolecular systems also can be tailored and controlled towards goal-optimizing. Here information flow, memory, adaptation and/or responsiveness are emergent properties in focus of contemporary research in complex nanomaterials frontiers In deed, the above presented paradigm shift concerning nucleation/crystallization and aggregation in complex, nanosized, materials bears a great potential for application in problems of self-organization for self-replicating systems as well as a variety of aggregation processes which involve auto-catalytic steps $[17,18]$ that are essential in the manufacturing of a novel class materials communally known as 'smart materials'. Recent advances are some novel ideas are presented and reviewed in [20] and the state of the art can be found in [19]. But, equally importantly, the above line of thinking has influenced, in the genuine interdisciplinary fashion that Grégoire Nicolis always taught, developments in another field, that of biological aggregation as we have just mentioned above and which we shall now turn.

3.2. Coordinated motion and decision making in the collective dynamics of social animals

In living systems, aggregation is defined as a higher temporal and spatial density of individuals in some part of space as compared to the surrounding area [21]. This ubiquitous phenomenon can be observed in many species, from bacteria to higher vertebrates, in response to environmental heterogeneities, but also to interactions between individuals [24] that are at the origin of positive feedbacks. In this sense, aggregation is a major manifestation of selforganisation in biology. A most simple scenario is provided when individuals from a group may gather on a single patch. In this case, the evolution in time of aggregated individuals $X$ depends on the rate of joining the patch $\mu$, which is proportional to the number of individuals outside, and on the rate of leaving the patch which decreases nonlinearly with the number of individuals already in this patch. Experiments show [22] that this leaving rate decreases with the number of individuals already present in the patch in a nonlinear fashion. The particular forms chosen here is

$$
\Xi(X)=\frac{\theta}{K^{\ell}+X^{\ell}}
$$

where $\theta$ is the individual probability to leave the patch and $K$ and $\ell$ are parameters related to how individuals respond to each other. The evolution equation of the number of individuals on the patch reads thus

$$
\frac{d X}{d t}=\mu N_{\mathrm{ext}}-\frac{\theta X}{K^{\ell}+X^{\ell}}
$$


where $N_{\text {ext }}$ is the number of individuals outside the patch $\left(N_{\text {ext }}=N-X\right)$. While relatively simple, the model defined by equation $1 b$ has up to three steady-states and the system displays hysteretic behaviour (Figure 6) against the parameter $\theta / \mu$. $\theta$ being the individual leaving rate, it actually depends on such parameters as the temperature, the relative humidity or the intensity of the light. Performing experiments with controlled changes on these parameters would undoubtedly assess the degree at which these states are stable against environmental changes and highlight Historydependent phenomena. When now confronted to different patches (be they food sources or shelters during resting times [25]), individuals may choose to gather on a unique patch or, on the contrary distribute more or less homogeneously in the patchy environment. These different outcomes are dependent on individual preferences but also on the strength of interactions between individuals and on the number of individuals involved. Such collective processes have been extensively studied at the species level [24], where individuals are considered identical but mixed species associations, while being frequent [23, 2628], did not received much attention. In order to study such systems, we augment Equation 1a to take into account the interattraction between two subgroups and the finite number of individuals that a patch is able to contain. The rates at which an individual from respectively subgroups $X$ and $Y$ leaves the patch $j$ for the patch $i$ can be written as

$$
\begin{aligned}
\Xi_{j i}\left(X_{j}, Y_{j}\right) & =\frac{\theta / S\left(X_{i}+Y_{i}\right)}{K^{\ell}+\left(\beta_{x} Y_{j}+X_{j}\right)^{\ell}}, \\
\Upsilon_{j i}\left(X_{j}, Y_{j}\right) & =\frac{\theta / S\left(X_{i}+Y_{i}\right)}{K^{\ell}+\left(\beta_{y} X_{j}+Y_{j}\right)^{\ell}}, \\
i, j & =1, m, i \neq j
\end{aligned}
$$

being understood that we neglect the outside world and that individuals "jump" from one patch to the other. Here, $\beta_{x}, \beta_{y}$ are interattraction parameters between the two different sub-groups and $\theta$ is the maximal speed at which individuals are joining and leaving the patches, modulated by its carrying capacity $S$ and its state of occupation.
The functions implicitly impose that the total population is smaller than or equal to the sum of the carrying capacities of the patches (e.g, total population $\leq m S$ ). The total fluxes of $X$ and $Y$ individuals between $j$ and $i$ are thus $\Xi_{j i} X_{j}$ and $\Upsilon_{j i} Y_{j}$ respectively. In the sequel, we will choose $\ell=2$ as the Hill exponent parameter, $N_{x}=N_{y}=N$ (i.e. subgroup populations of equal size) and $m=2$ (i.e. two patches). After nondimensionalization, the full model reduces to

$$
\begin{aligned}
\frac{d x_{i}}{d t} & =-\frac{x_{i}\left(1-\frac{1}{s}\left(x_{j}+y_{j}\right)\right)}{k^{2}+\left(\beta_{x} y_{i}+x_{i}\right)^{2}}+\frac{x_{j}\left(1-\frac{1}{s}\left(x_{i}+y_{i}\right)\right)}{k^{2}+\left(\beta_{x} y_{j}+x_{j}\right)^{2}} \\
\frac{d y_{i}}{d t} & =-\frac{y_{i}\left(1-\frac{1}{s}\left(x_{j}+y_{j}\right)\right)}{k^{2}+\left(\beta_{y} x_{i}+y_{i}\right)^{2}}+\frac{y_{j}\left(1-\frac{1}{s}\left(x_{i}+y_{i}\right)\right)}{k^{2}+\left(\beta_{y} x_{j}+y_{j}\right)^{2}} \\
i, j & =1,2, i \neq j
\end{aligned}
$$

where $x_{i}=X_{i} / N, y_{i}=Y_{i} / N, s=S / N$, $k=K / N$ and $t=\theta T / N^{2}$. We notice that $d x_{1} / d t+d x_{2} / d t=0 \quad$ and $\quad d y_{1} / d t+d y_{2} / d t=0$ reflecting that throughout the process the numbers of individuals are conserved $\left(x_{1}+x_{2}=1\right.$, $\left.y_{1}+y_{2}=1\right)$. In the case of a symmetrical case $\beta_{x}=\beta_{y}=\beta$, where interattraction acts in the same way for the two sub-groups, Equations (3) possess four types of solutions (see [29] for details):

- a homogeneous solution (referred to in the sequel as dispersion) defined by all four variables being equal

- four semi-homogeneous solutions defined by $x_{1}=y_{1}$ (referred to in the sequel as aggregation) and by $x_{1}=y_{2}$ (referred to in the sequel as segregation)

- four inhomogeneous solutions defined by $x_{1} \neq x_{2} \neq y_{1} \neq y_{2}$.

Figure 7a shows a bifurcation diagram of the steady solutions of Equations (3) for a particular value of $\beta$ and $s$. As noticed, for increasing values of $\beta$, the system switches from a state of segregation, where each sub-group aggregates under one shelter, to a dispersion state, where individuals from each sub-group are split between the two shelters, to a state of aggregation, where 


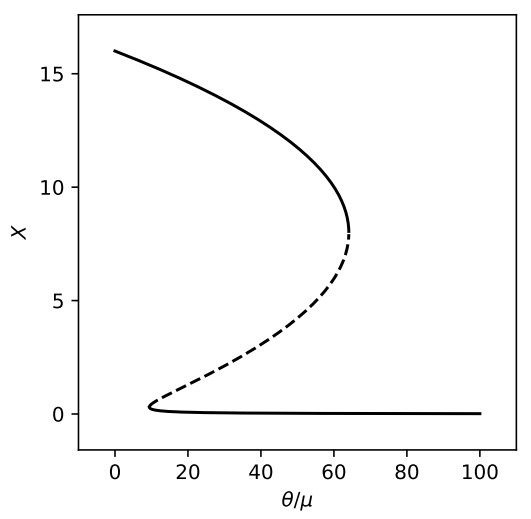

FIG. 6. Bifurcation diagrams of the steady states of $x_{1, s}$ of model (1b) as a function of parameter $\theta / \mu$ for $\ell=2.5, K=0.3$ and $N=16$

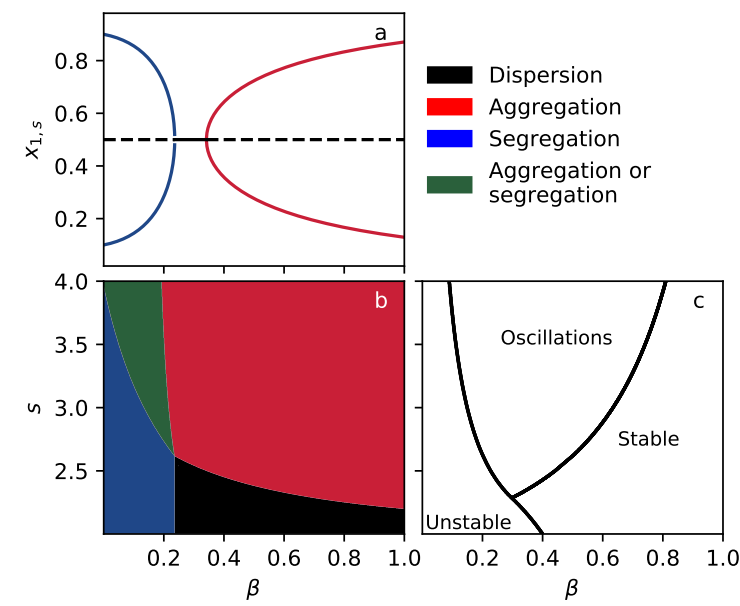

FIG. 7. (color online) Bifurcation diagrams of the steady states of $x_{1, s}$ of model (3) as a function of parameter $\beta$ for $s=2.5$ and $k=0.5$ (a). State diagram of the type of existing solutions as a function of $\beta$ and $s$ for $k=0.3$ (b).Stability of the homogeneous state of model (3) in the case of asymmetrical case $\beta_{x}=\beta$ and $\beta_{y}=0$ as a function of $\beta$ and $s$ for $k=0.3$ (c)

all individuals from each sub-group select one shelter. Figure $7 \mathrm{~b}$ shows a state diagram against parameters $s$ and $\beta$ for a particular value of $k$ where all the available states are available. The asymmetrical case $\beta_{x}=\beta, \beta_{y}=0$ where one sub-group is attracted to the other $\beta_{x}=\beta$ while the other is not $\beta_{y}=0$ is not fully accessible analytically, except for the homogeneous solution. Still, the nature of the solutions remains basically the same as in the previous case. For some range of parameter values one also has the possibility of oscillatory behaviour around the homogeneous state (Figure 7c).

All in all, the model depicted by Equations (3) shows non-trivial features like different aggregation patterns and coexistence between them. Of special interest is the state of segregation that is not induced by any agonistic behaviours but only by the finite size of each shelter, the individuals of the two subgroups interacting the same way.

\section{Outlook \& future fruits}

The prospect of incorporating historicity in complex system science has been heralded in Grégiore Nicolis work [35] in relation of the role of underlying bifurcation and hysteretic scenarii inherent in their nonlinear dynamical laws of evolution. That is to say that understanding how minute causes affect History is the key to understanding how societies are able to shift to other modes of organization. So naturally arises the question for future research [20]: is History also a core concept essential to simpler organisms or even 'smart' adaptive materials?

We envision that for the future, historydependent behaviours of complex aggregating systems can be highlighted thanks to the use of models and specifically designed experimental setups. We can already see how common similar features like displaying 'social' behavior during complex and directed aggregation [34] or certain propensities for self-organization can be influenced by embedded sensors and actuators, be it material or biologically based. Since complex systems are able to adopt multiple behavioral patterns depending on their initial state and constraints during their evolution one can to assess the stability of these patterns against environmental variations (hysteretic behaviors). Furthermore, a pattern being maintained in these changing conditions signals the existence of a collective memory - the principal manifestation of 
the History of the complex system. Our keystone hypothesis is that since History is encoded at the level of chemical/physical heterogeneities resulting from individual activities can also be utilized and directed towards specific goals. At this stage of development we are deeply motivated to further investigating the dynamics of decision making [32, 33] in complex systems. We strongly believe that pursuing the study of the dynamics in biological information processing is a rich, yet vast, area where insights from biology can influence and enrich the development of complex system science and vice versa, in a true interdisciplinary fashion faithful to the spirit of its founders.

\section{Acknowledgements}

We wish to thank the organizers Professors Angela De Sanctis and Anastasios Bountis for their invitation and kind hospitality. VB wants to extend his thanks to Dr. Pier Francesco Moretti of Italy's CNR for sharing ideas, resources and insights and for exciting discussions on Foresight's STEM materials initiative. And last but not least we wish to express our gratitude and affection to Grégoire's chief collaborator, his 'stable attractor', and life-long partner, his dear wife, Catherine (Kate) Nicolis.

\section{References}

[1] Ilya Prigogine, Nobel Lecture, December 8, 1977, "Time, Structure and Fluctuations". https://www.nobelprize.org/prizes/chemistry/ 1977/prigogine/lecture/

[2] P. Gaspard, G. Nicolis. What Can We Learn From Homoclinic Orbits in Chaotic Dynamics? J. Stat. Phys. 31, 499-518 (1983).

[3] G. Nicolis. The Many Facets of Complexity. In: Complexity Science. (World Scientific, Singapore, 2019). Pp. 3-30.

[4] Map of Complexity Science. Created by Hiroki Sayama, D.Sc., Collective Dynamics of Complex Systems (CoCo) Research Group at Binghamton University, State University of New York, "creative commons license".

[5] C. Nicolis. Solar variability and stochastic effects on climate. Sol. Phys. 74, 473-478 (1981).

[6] C. Rouvas-Nicolis, G. Nicolis.Stochastic resonance. Scholarpedia. 2(11), 1474, (2007).

[7] V. Basios, G-L. Forti, G. Nicolis. Symbolic dynamics generated by a combination of graphs. Int. J. of Bifurcation and Chaos. 18, 2265-2274 (2008).

[8] G. Nicolis, C. Nicolis, J.S. Nicolis . Chaotic dynamics, Markov partitions, and Zipf's law. J. Stat. Phys. 54, 915-924 (1989).

[9] P. Gaspard. Chaos, Scattering, and Statistical Mechanics. Nonlinear Science Series No. 9. (Cambridge University Press, 1998).

[10] J.J. Kozak, V. Basios, G. Nicolis. Geometrical effects in protein nucleation. Biophysical
Chemistry. 105, 495-501 (2003).

[11] G. Nicolis, C. Nicolis. Kinetics of phase transitions in the presence of an intermediate metastable state: A generic model. Physica A. 351(1), 22-39 (2005).

[12] J.F. Lutsko, G. Nicolis. Theoretical evidence for a dense fluid precursor to crystallization. Phys. Rev. Lett. 96, 46102 (2006).

[13] V. Basios, J. Lutsko, G. Nicolis, D. Maes, C. Kirschhock. a new paradigm of crystallization arising from non-standard nucleation pathways. Microgravity Science \& Technology. 21, 47-51, (2009).

[14] Kinetics and Thermodynamics of Multistep Nucleation and Self-Assembly in Nanoscale Materials. Eds. G. Nicolis, D. Maes. Advances in Chemical Physics. Book 324. (2012).

[15] J.F. Lutsko, V. Basios, G. Nicolis, T.P. Caremans, A. Aerts, J.A. Martens, C.E.A. Kirschhock, T.S. van Erp. Kinetics of intermediate-mediated self-assembly in nanosized materials: A generic model. J. Chem. Phys. 132, 164701 (2010).

[16] A. Buffin, S. Goldman, J-L. Deneubourg. Collective regulatory stock management and spatiotemporal dynamics of the food flow in ants. The FASEB Journal. 26, 2725-2733 (2012).

[17] S. Maiti, I. Fortunati, C. Ferrante, P. Scrimin, L.J. Prins. Dissipative self-assembly of vesicular nanoreactors. Nature Chemistry. 8, 725-731 (2016). 
[18] I. Colomer, S.M. Morrow, S.P. Fletcher. A transient self-assembling self-replicator. Nature Communications. 9, 2239 (2018).

[19] J.F. Lutsko. How crystals form: A theory of nucleation pathways. Science Advances. 5, eaav7399 (2019).

[20] P-F. Moretti, B. A. Grzybowski, V. Basios, E. Fortunato, M-S, Diez, O. Speck, R. Martins. STEM Materials: a new frontier for an intelligent sustainable world. BioMed Central, BMC Material, (in press) (2019).

[21] T.R.E. Southwood. Ecological Methods With Particular Reference To The Study Of Insect Populations. (Chapman And Hall, London, 1966).

[22] J. Halloy et al. Social integration of robots into groups of cockroaches to control self-organized choices. Science. 318, 1155-1158 (2007).

[23] J. Boulay et al. Mixed-species aggregations in arthropods. Insect Science. 26, 2-19 (2019).

[24] J. Krause, G.D. Ruxton. Living in groups. Oxford University Press, 2002).

[25] C. Rivault, A. Cloarec. Cockroach aggregation: Discrimination between strain odours inblattella germanica. Animal Behaviour. 55(1), 177-184 (1998).

[26] E.V.A. Stensland, A. Angerbjörn, P.E.R. Berggren. Mixed species groups in mammals. Mammal Review. 33(3-4), 205-223 (2003).

[27] D.R. Farine et al. Collective decision making and social interaction rules in mixed-species flocks of songbirds. Animal behaviour. 95, 173-182 (2014).

[28] B. Sauphanor, F. Sureau. Aggregation behaviour and interspecific relationships in Dermaptera. Oecologia. 96(3), 360-364 (1993).

[29] S.C. Nicolis, J. Halloy, J.-L. Deneubourg. Transition between Segregation and Aggregation: The Role of Environmental Constraints. Scientific Reports. 6, 32703 (2016).

[30] J.S. Nicolis, G. Nicolis, V. Basios. Chaos, information processing and paradoxical games. (World Scientific. Singapore, 2014).

[31] B. Vasileios, Y.-P. Gunji. Chaotic Dynamics in Biological Information Processing: Revisiting and Revealing its Logic (a mini-review). Opera Physiologica and Medica, OM\&P. 3(1), 1-13 (2017).

[32] Y-P. Gunji et al. Inverse Bayesian inference in swarming behaviour of soldier crabs. J. Philosophical Transactions of the Royal Society A. 376, 20170370 (2018).

[33] G. Nicolis, S.C. Nicolis. Probabilistic Network Approach to Decision-Making. Open Systems \& Information Dynamics. 22, 1550012 (2015).

[34] V. Basios, S. C. Nicolis, J.-L. Deneubourg. Coordinated aggregation in complex systems: an interdisciplinary approach. Eur. Phys. J. Special Topics. 225, 1143 (2016)

\section{Books by Grégoire Nicolis}

[35] G. Nicolis, C. Nicolis. Foundations of Complex Systems: Nonlinear Dynamics, Statistical Physics, Information and Prediction. 2nd edition. (World Scientific, Singapore, 2012).

[36] G. Nicolis. Introduction to Nonlinear Science. (Cambridge University Press, Cambridge, 1995).

[37] G. Nicolis, I. Prigogine. Exploring Complexity. (Freeman, New York, 1989).

[38] G. Nicolis , I. Prigogine. A la rencontre $d u$ complexe. (Presses universitaires de France, 1992).

[39] G. Nicolis, I. Prigogine. Self-Organization in Nonequilibrium Systems. (Wiley-Interscience, New York, 1977).

[40] S. Wuppuluri, F.A. Doria. Unravelling Complexity: The Life and Work of Gregory Chaitin. (World Scientific, Singapore, 2020).

Selected Links.

[41] http://homepages.ulb.ac.be/ gaspard/

[42] https://www2.ulb.ac.be/sciences/nlpc/ adewit.html

[43] http://homepages.ulb.ac.be/ jldeneub/

[44] http://www.scholarpedia.org/article/ Complex_systems 